\title{
Fibulin-5 downregulates Ki-67 and inhibits proliferation and invasion of breast cancer cells
}

\author{
YAMINA MOHAMEDI ${ }^{1,5}$, TANIA FONTANIL ${ }^{1,5}$, LAURA SOLARES ${ }^{1,5}$, OLIVIA GARCIA-SUÁREZ ${ }^{2,5}$, \\ JORGE GARCÍA-PIQUERAS ${ }^{2,5}$, JOSE A. VEGA ${ }^{2,4,5}$, SANTIAGO CAL ${ }^{1,5}$ and ALVARO J. OBAYA ${ }^{3,5}$
}

\author{
Departments of ${ }^{1}$ Biochemistry and Molecular Biology, ${ }^{2}$ Morphology and Cellular Biology and \\ ${ }^{3}$ Functional Biology-Physiology, Faculty of Medicine, University of Oviedo, Oviedo, Spain; ${ }^{4}$ Faculty of Health Sciences, \\ Autonomous University of Chile, Chile; ${ }^{5}$ University Institute of Oncology of Principado de Asturias (IUOPA), Oviedo, Spain
}

Received November 12, 2015; Accepted December 8, 2015

DOI: 10.3892/ijo.2016.3394

\begin{abstract}
Fibulins not only function as molecular bridges within the cellular microenvironment but also influence cell behavior. Thus, fibulins may contribute to create a permissive microenvironment for tumor growth but can also stimulate different mechanisms that may impede tumor progression. This is the case with Fibulin-5, which has been shown to display both tumor-promoting and tumor-protective functions by mechanisms that are not totally defined. We show new evidence on the tumor-protective functions displayed by Fibulin-5 in MCF-7, T47D and MDA-MB-231 breast cancer cells including the inhibition of invasion and proliferation capacity and hampering the ability to form mammospheres. Reduction in the level of phosphorylation of Ser residues involved in the nuclear translocation of $\beta$-catenin may underlie these antitumor effects. We also found that Fibulin-5 reduces the level of expression of Ki-67, a nuclear protein associated with cell proliferation. Moreover, reduction in Fibulin-5 expression corresponds to an increase of $\mathrm{Ki}-67$ detection in breast tissue samples. Overall, our data provide new insights into the influence of Fibulin-5 to modify breast cancer cell behavior and contribute to better understand the connections between fibulins and cancer.
\end{abstract}

\section{Introduction}

Fibulin-5, also known as DANCE, EVEC or UP50, is a 66-kDa secreted glycoprotein belonging to the fibulin family, whose members play essential roles in growth and tissue development

Correspondence to: Dr Alvaro J. Obaya, Departamento de Biología Funcional, Area de Fisiología, Facultad de Medicina, Universidad de Oviedo C/Julian Clavería, s/n. 33006, Oviedo-Asturias, Spain E-mail: ajobaya@uniovi.es

Abbreviations: DAPI, 4'-6'-diamino-2-phenylindole hydrochloride; ECM, extracellular matrix; EMT, epithelial-mensenchymal transition; $\mathrm{kDa}$, kilodaltons; RGD, arginine-glycine-aspartic acid

Key words: tumor biology, breast cancer, cell proliferation, cell migration, $\beta$-catenin, mammospheres formation
$(1,2)$. Fibulins can act as molecular bridges within the ECM, and as mediators of cellular processes including cell adhesion and motility, cell-cell and cell-ECM communications and elastogenesis. In particular, Fibulin-5 is an elastogenic short fibulin that interacts with different ECM proteins such as tropoelastin, fibrillin-1, EMILIN-1, lysyl oxidase-like I or apolipoprotein A $(3,4)$. It is also noteworthy that Fibulin-5 is the only member of the family that contains an RGD motif within its structure, which allows its interaction with a subset of integrins (5). Functional importance of Fibulin-5 is also highlighted when performing the phenotypic characterization of the mice deficient in Fbln5, the gene coding for murine fibulin-5 (6). Although these mice live through adulthood, they exhibit anatomical abnormalities related to elastic fiber assembly with resulting emphysematous lungs, vascular disorders and cutis laxa. Notably, mutations indentified in human FBLN5 gene have been also associated to age-related macular degeneration and cutix laxa $(7,8)$.

In addition to their role in tissue architecture and function, fibulins have also been involved in tumorigenesis. Thus, different fibulins have been found overexpressed or downregulated in tumor or stromal cells showing both oncogenic and tumor-protective properties $(9,10)$. In this regard, Fibulin-5 elicits tumor-promoting effects in nasopharyngeal carcinoma as its overexpression significantly correlates with advanced tumor metastasis (11); and Fibulin-5 is also involved in pulmonary metastasis of a rat model of renal cell carcinoma (12). Moreover, Fibulin-5 mediates EMT in mouse 4T1 breast tumor cells (13), and in human HeLa cervical cancer cells (14). By contrast, a growing number of studies highlight the antitumor effects caused by Fibulin-5. For instance, Fibulin-5 inhibits invasion and proliferation of the human bladder cancer cell line 5637 (15), and hampers cancer invasion and tumor metastasis of lung cancer cell lines $(16,17)$. Furthermore, Fibulin-5 can inhibit angiogenesis induced by HT1080 (18) and MCA102 (19) fibrosarcoma cell lines. Additionally, FBLN5 gene has been found epigenetically silenced in prostate (20) and lung cancer (16), which reinforces the tumor-suppressive roles of Fibulin-5.

In the present study, we have employed well-known human breast cancer cell models to overexpress Fibulin- 5 and to investigate changes in cell phenotype using different cell-based 
approaches including cell proliferation, migration and invasion assays, as well as mammosphere formation. Overall, our data suggest that Fibulin-5 induces antitumor effects by suppression of $\beta$-catenin phosphorylation. Immunohistochemical analysis of tumor samples of breast cancer patients indicated that a high Fibulin-5 expression level is concomitant with a low expression of the proliferative marker Ki-67 (21), which suggest that Fibulin-5 may influence breast cancer cell proliferation.

\section{Materials and methods}

Cell culture and transfection. MCF-7, T47D and MDA-MB-231 breast cancer cells and A549 lung cancer cell lines were routinely maintained in Dulbecco's Modified Eagle's medium (DMEM) containing 10\% heat-inactivated fetal bovine serum and $50 \mu \mathrm{g} / \mathrm{ml}$ streptomycin and $100 \mathrm{U} / \mathrm{ml}$ penicillin (Life Technologies, Carlsbad, CA, USA). These cell lines were kindly provided by Dr Carlos López-Otín (Universidad de Oviedo). pcDNA3 plasmid containing full-length cDNA for FBLN5 (22) was kindly provided by Dr William P. Schiemann (Case Western Reserve University, Cleveland, OH, USA). This expression vector was transfected into cells using TransIT-X2 Dynamic Delivery System (Mirus Bio LLC, Madison, WI, USA) as recommended by the manufacturer. Cells stably expressing exogenous FBLN5 were selected in the presence of $500 \mu \mathrm{g} / \mathrm{ml} \mathrm{G} 418$ (Sigma-Aldrich). In all experiments cells transfected with an empty vector were employed as a control.

Western blot analysis and cell staining. Cell extracts were resolved by $10 \%$ polyacrylamide gel electrophoresis, transferred to a PVDF membrane and then probed with a rabbit anti-Fibulin-5 antibody (Origene Technologies Inc., Rockville, MD, USA). Rabbit anti- $\beta$-catenin antibodies were from Cell Signaling Technology (Danvers, MA, USA), rabbit anti-Ki-67 antibody was from Santa Cruz Biotechnology (Dallas, TX,USA) and mouse monoclonal anti- $\beta$-actin antibody (AC-40) was from Sigma-Aldrich Química S.A. (Madrid, Spain). Immunoreactive proteins were visualized using HPR-peroxidase labeled anti-rabbit antibody (Pierce, Dorchester, MA, USA). For immunocytochemical analysis, breast cancer cells expressing Fibulin-5 were fixed with 3.7 paraformaldehyde in phosphatebuffered saline buffer and cells were then blocked with $10 \%$ fetal bovine serum. To detect $\mathrm{Ki}-67$, blocked slides were incubated overnight with the H-300 antibody from Santa Cruz Biotechnologies, followed by 1-h incubation with a secondary Alexa 546-conjugated antibody (Life Technologies, Carlsbad, CA, USA). In all samples, DAPI was added at $100 \mathrm{ng} / \mathrm{ml}$ to visualize DNA in the cell nucleus. Images were obtained using a fluorescence microscope (Axiovert).

Cell proliferation assay. Cell proliferation was assayed using the CellTiter 96 Non-radiactive Cell Proliferation assay kit from Promega Biotech Ibérica SL (Madrid, Spain). MCF-7, T47D and MBA-MB-231 cells (5x10\%3ell) were seeded in 96-well plates and six replicates per condition. Cell proliferation rates were measured on four days using an automated microtiter plate reader Power WaveWS (Bio-Tek Instruments, Inc., Winooski, VT, USA). Additionally, cell proliferation was also estimated as an average of $\mathrm{Ki}$-67-positive nuclei in relation to the total number of nuclei per microscopic field $(n=4)(23)$.
Invasion assay. In vitro invasion potential was examined by using 24-well Matrigel-coated invasion chambers with an $8-\mu \mathrm{m}$ pore size (BD Biosciences, San Jose, CA, USA). To this end, $5 \times 10^{4} \mathrm{MCF}-7$ or T47D cells were allowed to migrate for $96 \mathrm{~h}$ using $10 \%$ fetal bovine serum as a chemoattractant. In the case of MDA-MB-231 cells, migration time was $24 \mathrm{~h}$. At least three independent experiments were made for each condition. Non-invading cells on the upper surface were removed from the chambers using a cotton swab and cells that reached the lower surface were fixed with $100 \%$ methanol and stained with $0.5 \%$ crystal violet in $2 \%$ ethanol. Cells were counted in four randomly selected microscopic fields.

Mammosphere assay. To evaluate mammosphere formation capacity of breast tumor cells overexpressing Fibulin-5, $4 \times 10^{4}$ MCF-7 and T47D cells were plated in 6-well ultralow attachments plates (Corning Costar, Corning, NY, USA) and grown in MammoCult Basal Medium (Stemcell Technologies, Vancouver, Canada) supplemented with 10\% MammoCult proliferation supplement, $4 \mu \mathrm{g} / \mathrm{ml}$ heparin and $0.5 \mu \mathrm{g} / \mathrm{ml}$ hydrocortisone. After 7 days, cells were collected and enzymatically dissociated as previously described (24). Then, individual dissociated cells were cultured in 96-well ultralow attachment (Corning Costar) at a density of 20 cells/well. Mammosphere formation was daily monitored to ensure that they derived from single cells. Number of mammospheres were determined after 7 days and mammospheres were again counted and graphically presented.

Migration assay. To evaluate the migratory capacity on ECM components, we employed the Radius ${ }^{\mathrm{TM}}$ 24-Well Cell Migration assay kit (Cell Biolabs Inc., San Diego, CA, USA), following the manufacturer' instructions. Briefly, $1.25 \times 10^{4}$ MCF-7 and MDA-MB-231 cells overexpressing Fibulin-5 or transfected with an empty vector were seeded on wells coated with fibronectin and type-I collagen. Migration was monitored for $12 \mathrm{~h}$ using a time-lapse Zeiss Axio Observer Microscopy. Experiments were performed in triplicates and covered area was quantified at different times using ImageJ software.

Human breast cancer tissue array. A breast cancer tissue array containing 78 samples of different types of human breast tumors and 3 normal tissues was obtained from the Institute of Oncology of Asturias Tumor Bank. Study subjects were newly diagnosed with breast cancer and histopathologically confirmed at the Hospital Universitario Central de Asturias (HUCA). Tumor-free samples were selected from healthy tissue areas and immediately frozen. Written informed consent was obtained from all patients prior to sample collection. The study was approved by the appropriate institutional review board according to national and EU guidelines. To perform the histological analysis, deparaffinized and rehydrated sections were processed for detection of Fibulin-5 and Ki-67 using the EnVision antibody complex kit (Dako Denmark A/S, Copenhagen, Denmark) following the manufacturer's recommendations. The rabbit anti-Fibulin-5 antibody (Abcam) was diluted 1:200 and the anti-Ki67 was a mouse monoclonal antibody (Dako, clone MIB-1) diluted 1:100. For control purposes, representative sections were processed as above but 
A

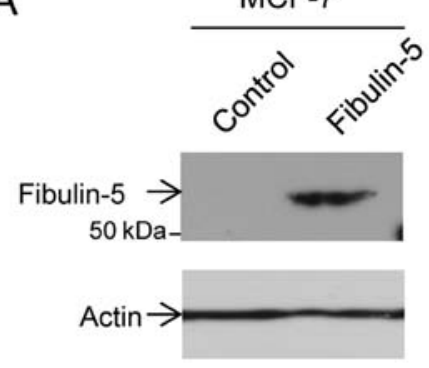

B

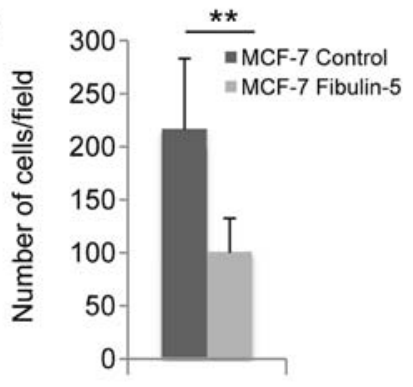

MCF-7

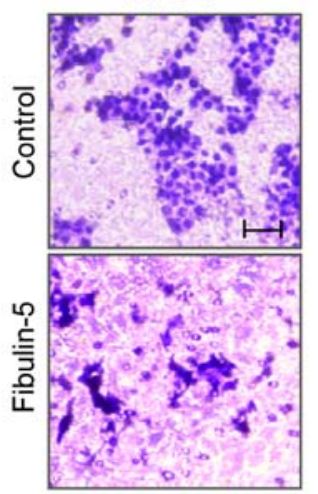

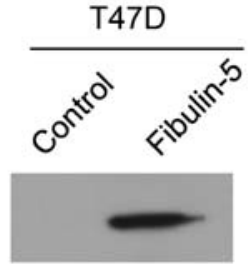
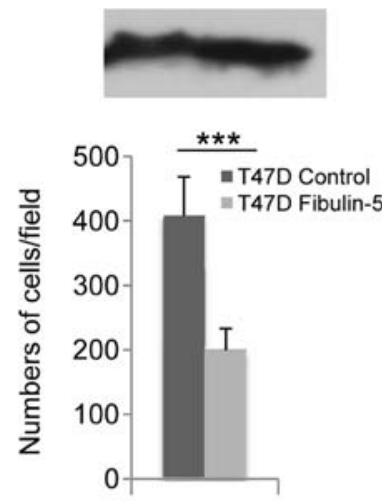

T47D

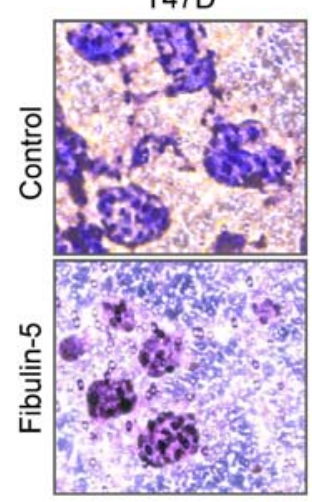

MDA-MB-231
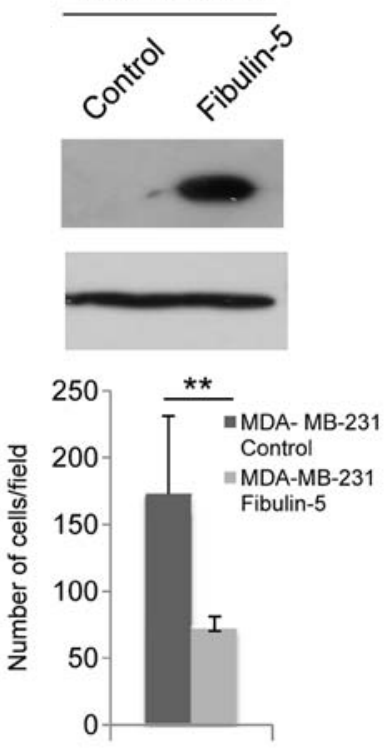

MDA-MB-231

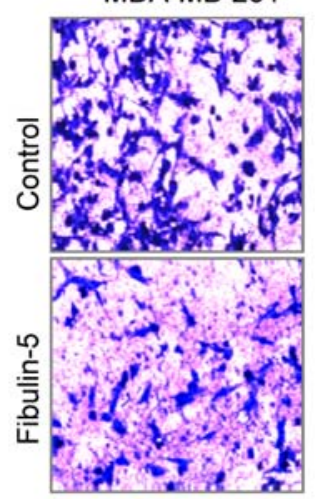

Figure 1. Expression of exogenous Fibulin-5 in breast cancer cells and invasion assay. (A) Western blot analysis of MCF-7, T47D and MDA-MB-231 cells extracts producing exogenous Fibulin-5. Actin was used as a loading control. Control, cells transfected with an empty vector. Molecular weight marker is indicated on the left. (B) Top, cell invasion assay using Matrigel-coated invasion chambers. Cells reached the lower surface of the membrane were counted and graphically represented. Bottom, representative microscopic pictures of the invasive cells. Scale bar, $50 \mu \mathrm{m}$.

rabbit or goat non-immune serum, or blocking buffer, were used instead of the primary antibodies. For simultaneous detection of Fibulin-5 and Ki-67 double immunofluorescence coupled to laser confocal microscope was used. Non-specific binding was reduced by incubation for 30 min with a solution of $1 \%$ bovine serum albumin in TBS (Tris-buffered saline buffer). Sections were incubated overnight, at $4^{\circ} \mathrm{C}$ in a humid chamber with a $1: 1$ mixture of anti-fibulin-5 and anti-Ki-67 antibodies, diluted 1:200 and 1:100, respectively. After rinsing with TBS, sections were incubated for $1 \mathrm{~h}$ with Alexa Fluor 488-conjugated goat anti-rabbit IgG (AbD Serotec, Kidlington, UK), diluted 1:1,000 in TBS containing $5 \%$ mouse serum (AbD Serotec), then rinsed again, and incubated for another hour with CyTM3-conjugated donkey anti-mouse antibody (Jackson ImmunoResearch) diluted 1:50 in TBS. Double fluorescence was detected using a Leica DMR-XA automatic fluorescence microscope (Photonic Microscopy Service, University of Oviedo) coupled with a Leica Confocal software, version 2.5 (Leica Microsystems, Wetzlar, Germany) and the images captured were processed using Image J version $1.43 \mathrm{~g}$ software, McMaster Biophotonics Facility (www.macbiophotonics.ca).
Survival analysis. To evaluate the effect of Fibulin-5 on breast cancer prognosis, a Kaplan-Meier log-rank test survival plot was performed using the data available at www.kmplot.com (affy ID 203088_at) (25).

Statistical analysis. To perform statistical analysis, we employed GraphPad Prism 5.0 software and data are presented as means \pm SE. Significant differences were determined with the Student-Welch t-test and P-values $<0.05$ were considered statistically significant (in the figures as: ${ }^{*} \mathrm{P}<0.05,{ }^{* *} \mathrm{P}<0.01$, $\left.{ }^{* * *} \mathrm{P}<0.005\right)$.

\section{Results}

Recombinant Fibulin-5 reduces breast cancer cell invasion. In the present study we wanted to examine if Fibulin-5 induces changes in the behavior of three commonly employed human breast cancer cells, the poorly invasive T47D and MCF-7 and the highly invasive MDA-MB-231 cell lines. Following transfection, production of recombinant Fibulin-5 was analyzed by western blot analysis using an anti-Fibulin- 5 antibody (Fig. 1A). An immunoreactive band corresponding to 
A

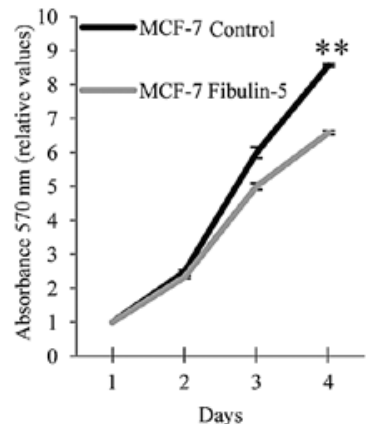

B

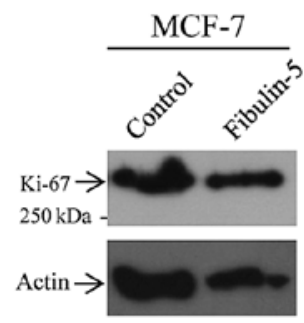

$\mathrm{C}$

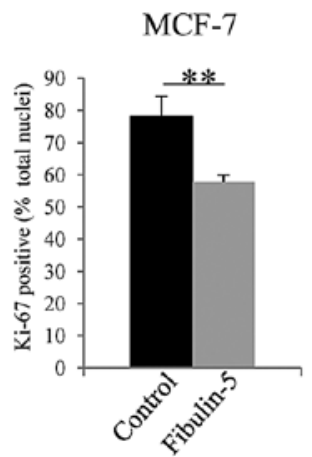

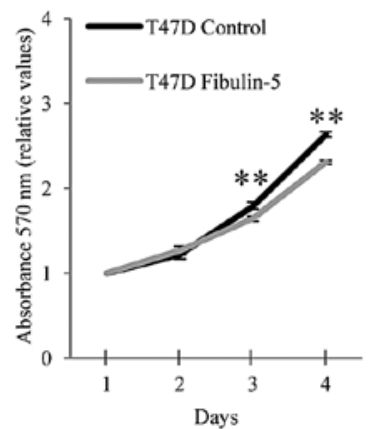
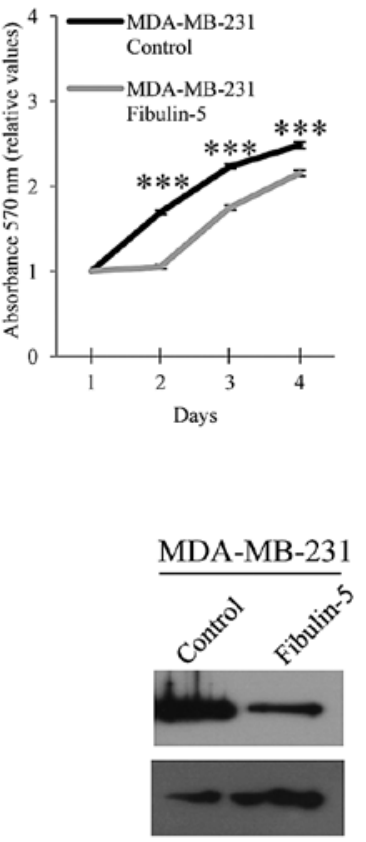

MDA-MB-231

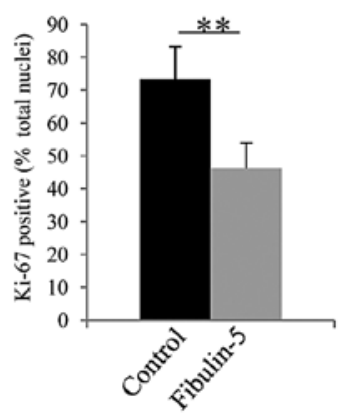

Figure 2. Effect of Fibulin-5 on breast cancer cell proliferation. (A) Cell proliferation values of MCF-7, T47D and MDA-MB-231 cells expressing exogenous Fibulin-5 and control cells were calculated on 4 consecutive days using the MTT assay. (B) Expression of the proliferation marker Ki-67 is reduced in the presence of Fibulin-5. Top, detection of Ki-67 by western blot analysis in MCF-7, T47D and MDA-MB-231 cell extracts. A significant reduction of Ki-67 expression is detected in cells overproducing Fibulin-5 in comparison with control cells. Molecular weight marker is indicated on the left. Actin was used as a loading control. (C) Graphical representation of Ki-67 positive cell nuclei compared to total number of nuclei $(n=4)$.

Fibulin-5 was detected in cell extracts containing Fibulin-5, but not in control cells transfected with an empty vector. To assess whether Fibulin-5 could affect cell invasion, cells were allowed to invade using Matrigel-coated invasion chambers (Fig. 2B). After 96 h, invasion of T47D and MCF-7 was analyzed with the finding that the presence of Fibulin-5 reduced the invasive potential of both cell lines in comparison with control cells $(45.5 \%$ reduction in the case of T47D cells; and $49.8 \%$ reduction in MCF-7 cells). MDA-MB-231 cells were allowed to invade for $24 \mathrm{~h}$ and cells producing recombinant Fibulin-5 showed $40.4 \%$ reduction in their invasive capacity when compared to control cells (Fig. 1B). These results suggest that Fibulin-5 strongly affects invasive capacity of breast cancer cells.

Fibulin-5 affects proliferation of breast cancer cells. To assess the potential effect of Fibulin-5 on breast cancer cell proliferation we performed two different approaches. First, we measured cell proliferation on 4 consecutive days employing a MTT assay. As shown in Fig. 2A, presence of Fibulin-5 reduced the number of T47D, MCF-7 and MDA-MB-231 proliferating cells. It is noteworthy that the highly invasive MDA-MB-231 cells seemed to be more affected than the other two since difference in cell proliferation between MDA-MB-231 cells expressing recombinant Fibulin-5 and control cells was already evident on the second day of the assay. Second approach was to examine the presence of the proliferation marker Ki-67 in Fibulin-5-transfected breast cancer cells. Western blot analysis showed that expression level of $\mathrm{Ki}-67$ was considerably reduced in breast cancer cell lines expressing Fibulin-5 when compared to control cells (Fig. 2B). Moreover, nuclear staining of the cells using an anti-Ki-67 antibody revealed that the number of nuclei showing $\mathrm{Ki}$-67-positive staining was lower in cells expressing recombinant Fibulin-5 than in control 

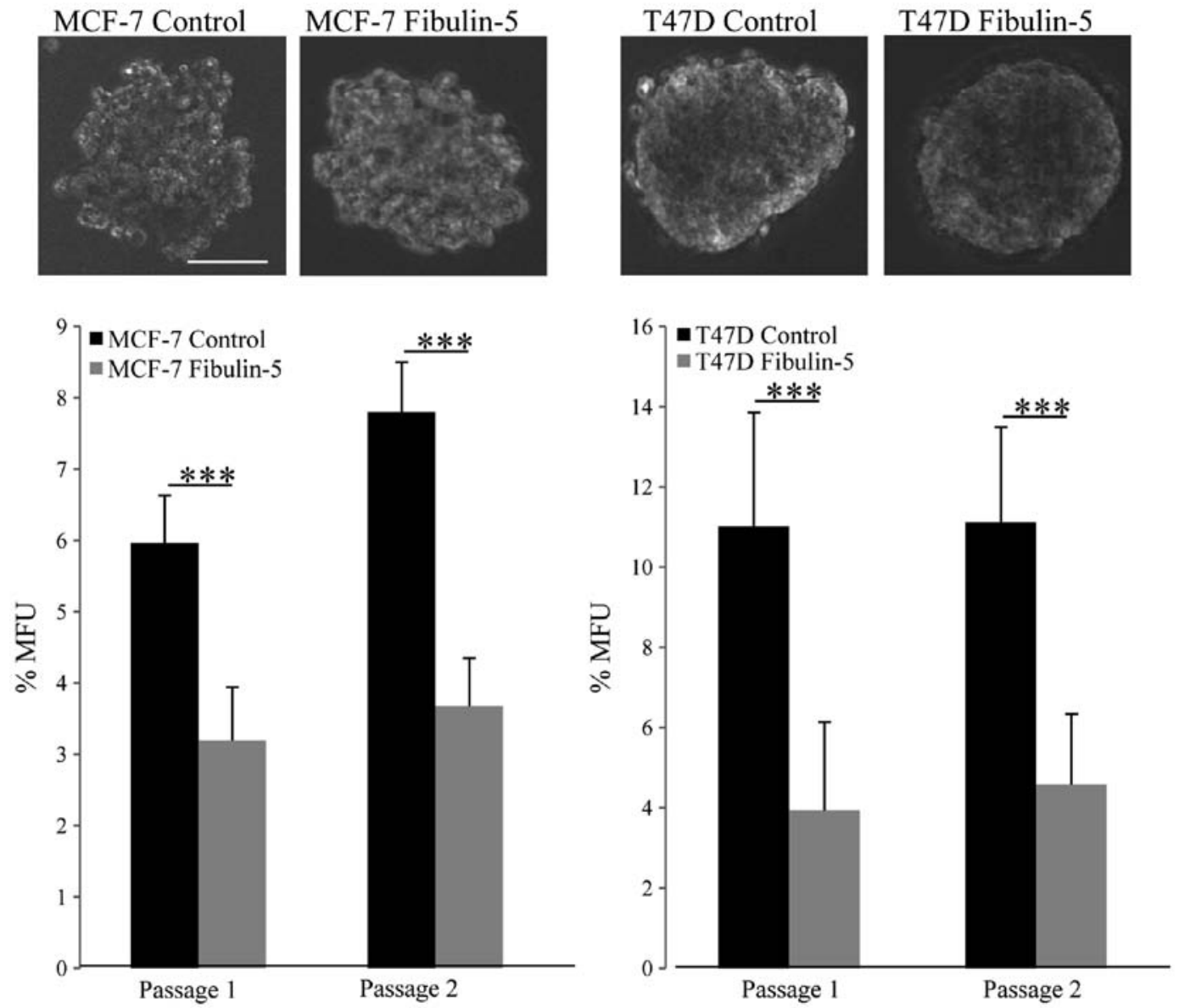

Figure 3. Fibulin-5 affects self-renewal of mammosphere forming units. Presence of Fibulin-5 does not seem to affect size or morphology of mammospheres derived from MCF-7 and T47D breast cancer cells (upper images, scale bar, $50 \mu \mathrm{m}$ ). However, a significant decrease in the number of MFU is observed in MCF-7 and T47D expressing Fibulin-5 in comparison to control cells in two consecutive passages.

cells. Thus, $\mathrm{Ki}-67-$ positive nuclei represented an average of 78 , 64 and 72\% in control MCF-7, T47D and MDA-MB-231 cells respectively, whereas 58,50 and $44 \%$ of total nuclei showed positive staining in cells expressing exogenous Fibulin-5 (Fig. 2B). These data indicate that Fibulin-5 has an effect on breast cancer cell proliferation.

Fibulin-5 decreases the number of mammosphere formation units. MCF-7 and T47D are breast tumor cell lines that show a strong ability to form mammospheres (26). To evaluate whether the presence of Fibulin-5 could alter this ability, we carried out mammosphere forming assays that showed that the presence of Fibulin-5 did not seem to affect the size or morphology of the mammospheres (Fig. 3). However, we found a significant decrease in the number of mammospheres derived from MCF-7 and T47D cells expressing recombinant Fibulin-5. Indeed, Fibulin-5 decreased $\sim 50 \%$ the mammosphere forming units (MFU) in the case of MCF-7 cells and $\sim 64 \%$ in the case of T47D cells in comparison with control cells and in two consecutive passages (Fig. 3). Overall, these data indicate that capacity for in vitro self-renewal of MCF-7 and T47D cells is constrained by the presence of Fibulin-5.

Effect of Fibulin-5 on breast cancer cell migration. Next, we wanted to explore the possibility that Fibulin-5 affected breast cancer cell migration on type-I collagen and fibronectin, two extracellular matrix proteins that influence cell adhesion and migration properties of both the poorly invasive MCF-7 cells and the highly invasive MDA-MB-231 cells (27). After 12 h, MCF-7 expressing Fibulin-5 and control MCF-7 cells did not show differences in cell migration using wells coated with type-I collagen or fibronectin (Fig. 4). MDA-MB-231 cells also showed no differences between cells expressing Fibulin-5 and control cells in wells coated with fibronectin (Fig. 4). However, presence of Fibulin-5 affected the migratory capacity of MDA-MB-231 cells in wells coated with type-I collagen (Fig. 5). Thus after 12 h, $87.7 \%$ of area was covered by control MDA-MB-231 cells while only $53.9 \%$ was covered by MDA-MB-231 cells expressing Fibulin-5 ( $\mathrm{P}<0.01)$. Differences in migration on type-I collagen were already evident at $4 \mathrm{~h}(48.2$ vs. $5.7 \% ; \mathrm{P}<0.01)$. These data indicate that Fibulin-5 may act as a modulator of metastatic breast cancer cell migration through type-I collagen.

Presence of Fibulin-5 inhibits $\beta$-catenin phosphorylation. Then we asked whether the functional changes due to Fibulin-5 could be attributed to alterations in $\beta$-catenin phosphorylation. Western blot analysis revealed a decrease in the phosphorylation levels of the $\beta$-catenin residues $\operatorname{Ser}^{552}$ and $\operatorname{Ser}^{675}$ in MCF-7 cells (Fig. 6). Attending to previous results on the effect of Fibulin-5 in Wnt// 3 -catenin signaling pathway in lung cancer (17), we evaluated whether the human lung carcinoma 

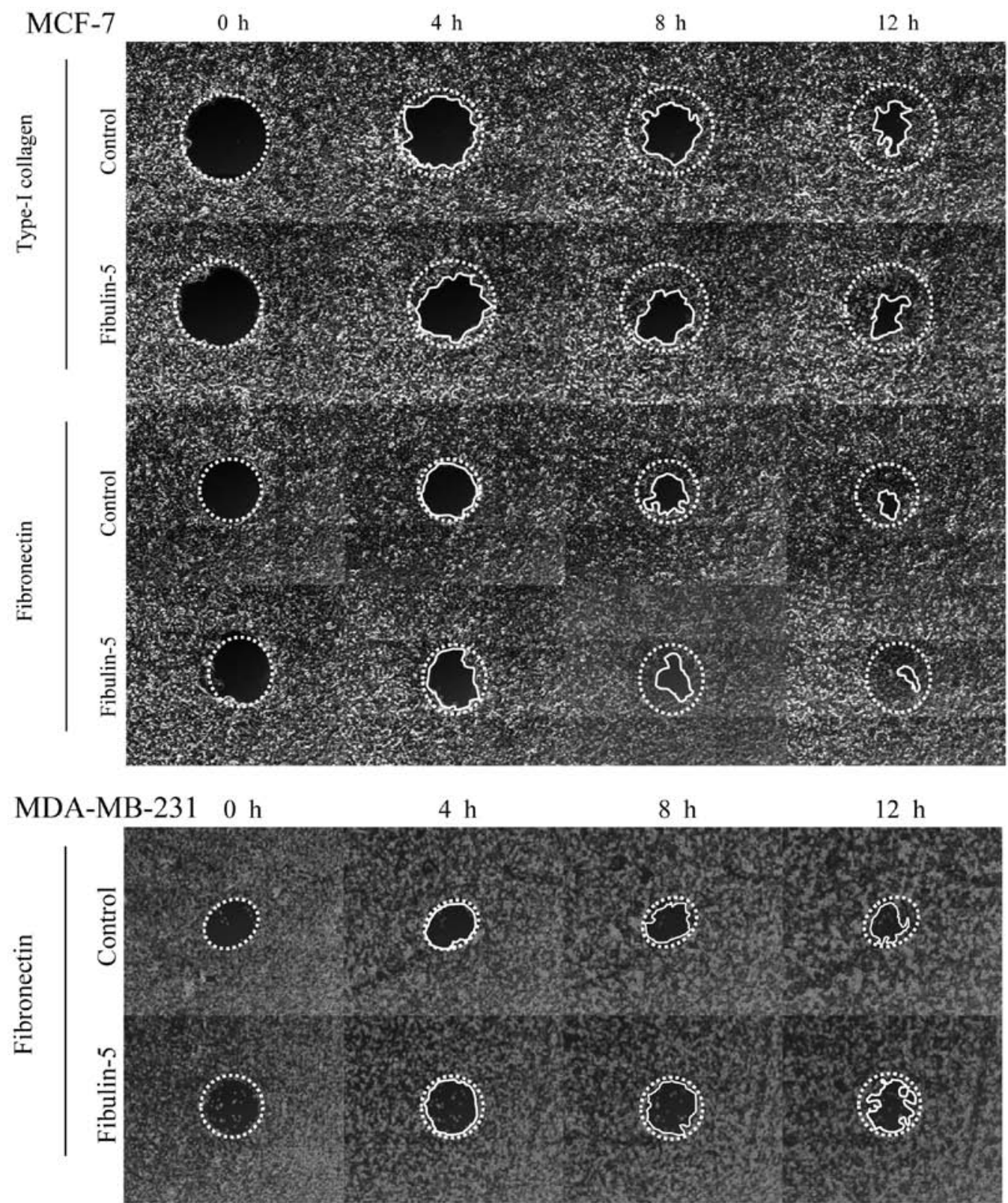

Figure 4. Upper panel, migratory capacity of MCF-7 cells expressing Fibulin-5 on type-I collagen and fibronectin. Lower panel, migratory MDA-MB-231 cells expressing Fibulin-5 on fibronectin. Cells transfected with an empty vector were employed as control in each case. Images were taken at the indicated times. Starting point is indicated with a thin dotted line and final point with a solid line.

cell line A549 undergoes the same effect, with the finding that Fibulin-5 also affects phosphorylation of $\operatorname{Ser}^{552}$ and $\operatorname{Ser}^{675}$ in this cell line (Fig. 6). Ser ${ }^{52}$ and $\operatorname{Ser}^{675}$ residues are involved in the translocation of $\beta$-catenin from cell-cell contacts into the nucleus. In consequence, decrease in $\beta$-catenin phosphorylation could underlie the changes observed in the phenotype of breast cancer cells employed in the present study.

Immunostaining of Fibulin-5 and Ki-67 in breast tumors. Taking into account our previous results on Fibulin-5 and Ki-67 in breast cancer cells, we next performed an immunohistochemical analysis to evaluate the simultaneous detection of Fibulin-5 and Ki-67 in breast cancer samples. To this end we employed a tissue array containing samples of the most common types of breast tumors as well as 3 normal breast tissues (Fig. 7). In normal samples, Fibulin-5 was present in both lobular and ductal epithelial cells, and the intensity of immunostaining varied among different segments of the organ. The density of $\mathrm{Ki}-67$ positive nuclei profiles was very low or undetectable in wide segments of the tissue. In tumor samples, immunoreactivity of Fibulin-5 was very low or absent in those samples showing medium or high expression of Ki-67 independently of tumor type (Fig. 7A). In ductal carcinoma, Fibulin-5 showed high immunoreactivity in the most advance stages of the disease. In parallel, the density of Ki-67 immunoreactive nuclei profiles decreased. By contrast, in lobular carcinoma, the expression of Fibulin-5 and Ki-67 showed an opposite evolution. Double immunofluorescence for simultaneous detection of Fibulin-5 and Ki-67 was used to ascertain these results (Fig. 7B). In a representative section of poorly differentiated ductal carcinoma, abundant tumor cells displaying strong Fibulin-5 immunofluorescence were found 

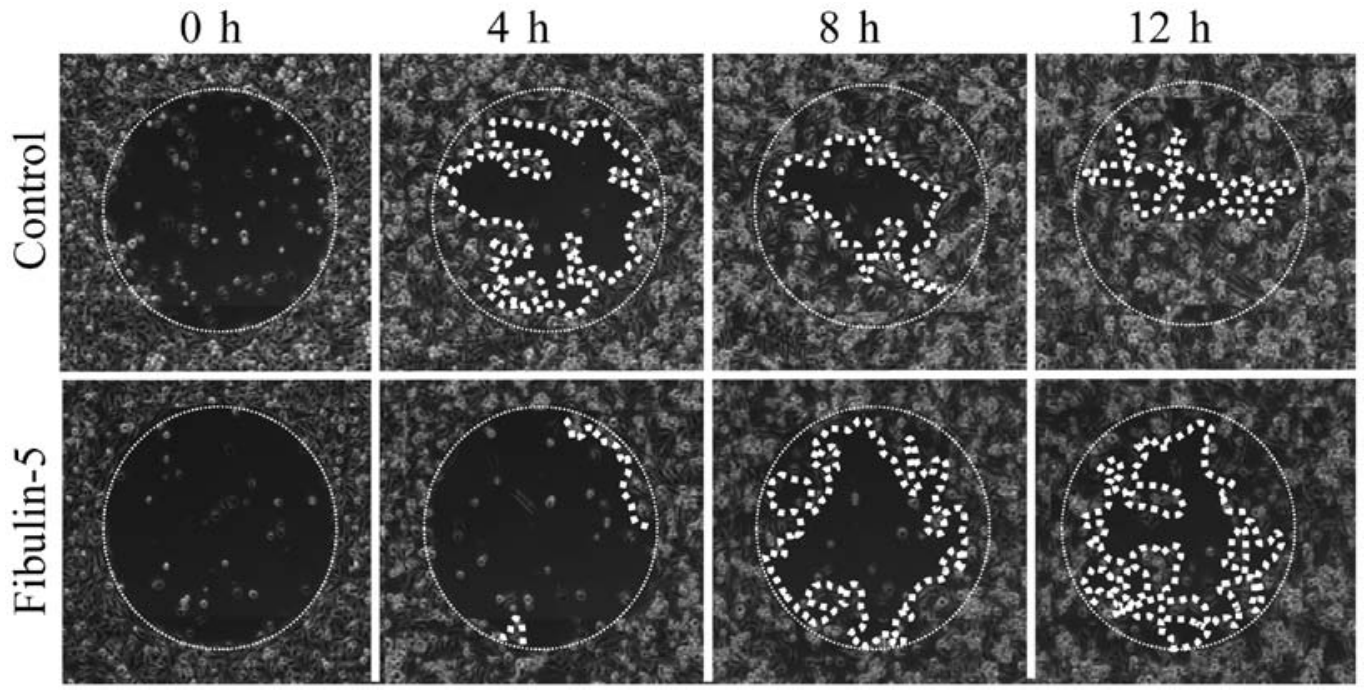

Figure 5. Fibulin-5 hinders migration of MDA-MB-231 cells on type-I collagen. MDA-MB-231 expressing Fibulin-5 were allowed to migrate on wells coated with type-I collagen. Cells transfected with an empty vector were employed as control. Images at $0 \mathrm{~h}$ (starting point, indicated with a thin dotted circle), 4,8 and $12 \mathrm{~h}$ (final point) are shown. Area covered by the cells at the different times is indicated with thick dotted line.
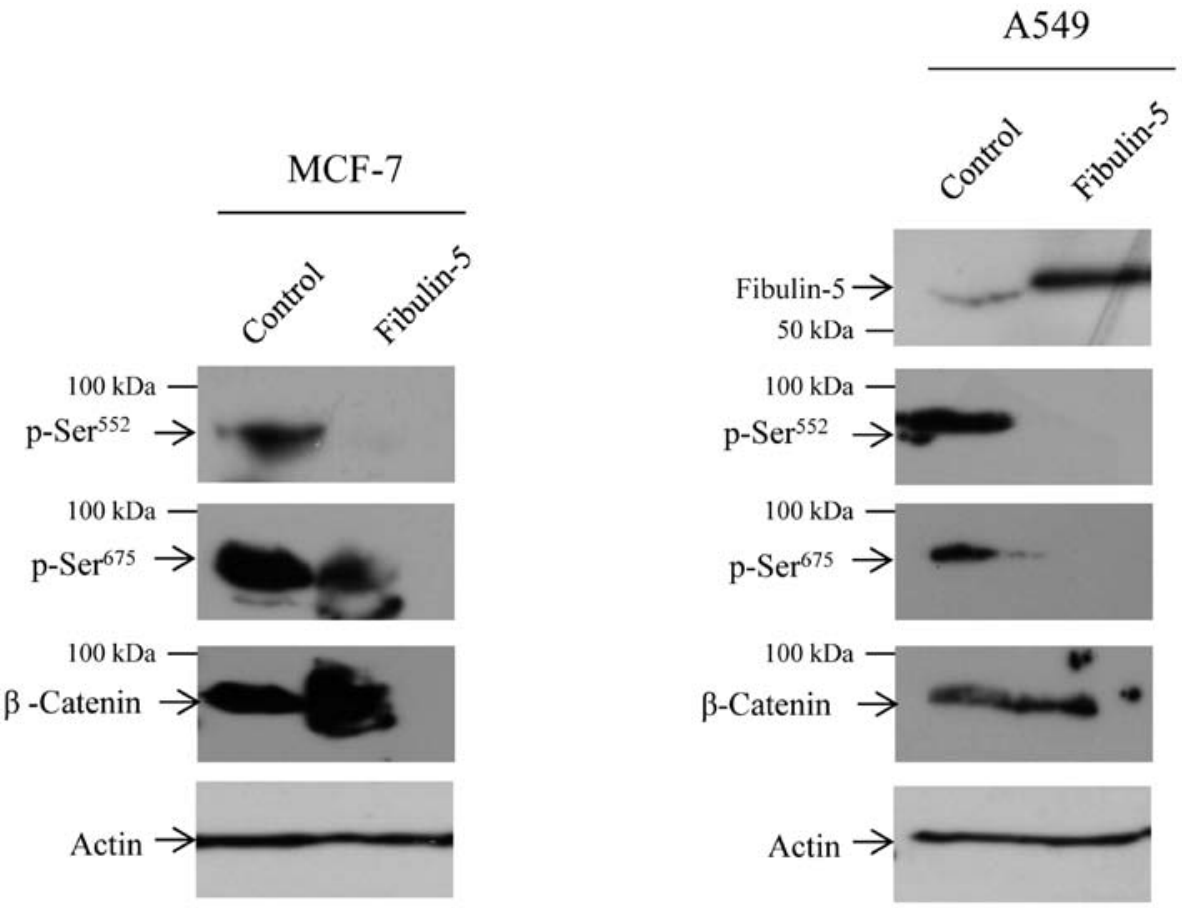

Figure 6. Fibulin-5 reduces phosphorylation levels of $\beta$-catenin. MCF-7 cells expressing exogenous Fibulin-5 show a reduction in phosphorylation levels in both $\mathrm{Ser}^{52}$ and $\mathrm{Ser}^{675}$, residues involved in the nuclear translocation of $\beta$-catenin. Following transfection, similar western blot analysis was performed in A549 cells that also exhibit an important decrease in the phosphorylation levels of these serine residues. Molecular weight markers are indicated on the left. Actin was employed as a loading control.

which were devoid of Ki-67 immunofluorescence. Conversely, in sections of poorly differentiated lobular carcinoma Fibulin-5 was absent in tumor cells while they showed numerous Ki-67 positive nuclei profiles. This analysis also indicated that Fibulin-5 was not colocalized with Ki-67 in most samples examined. Moreover, Kaplan-Meier analysis using the data available at www.kmplot.com revealed that breast cancer patients with high levels of Fibulin-5 showed better prognosis than patients expressing low levels (Fig. 7C). This analysis suggests that FBLN5 gene expression is associated with better outcome in breast cancer patients.

\section{Discussion}

The number of studies supporting the importance of fibulins in tumorigenesis has considerably grown in recent years (10). Role of these secreted glycoproteins in cancer is still controversial as they can induce both tumor-promoting 
A

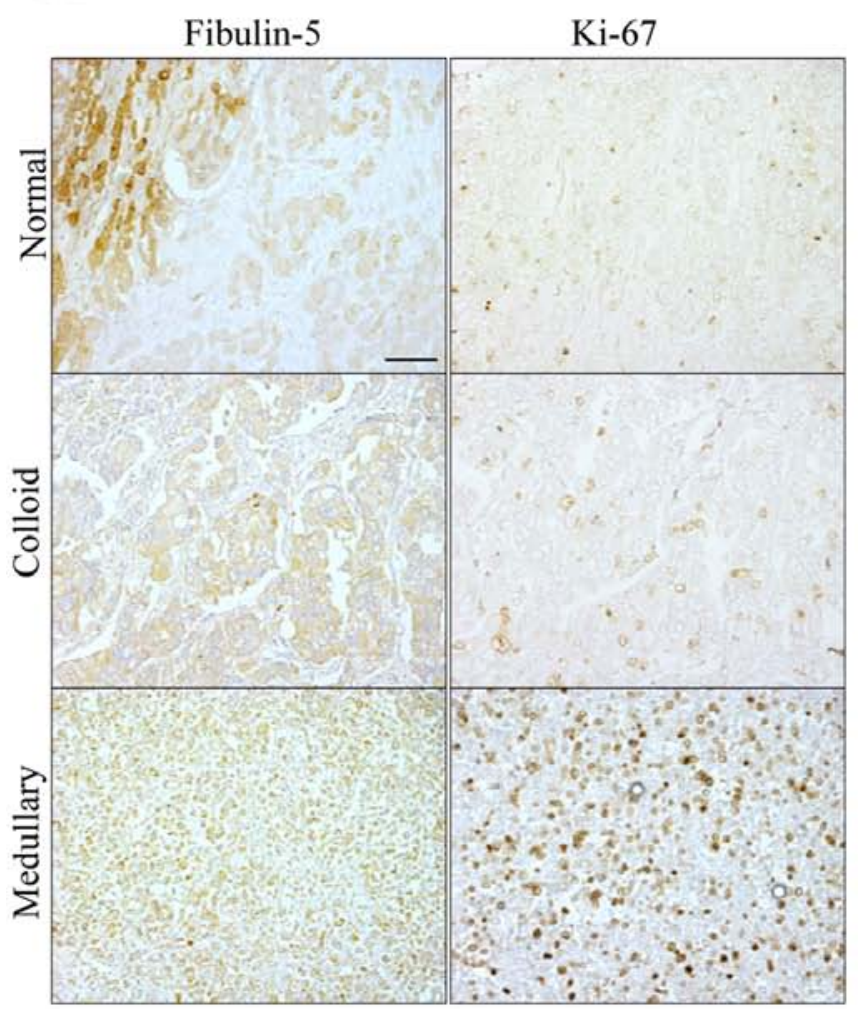

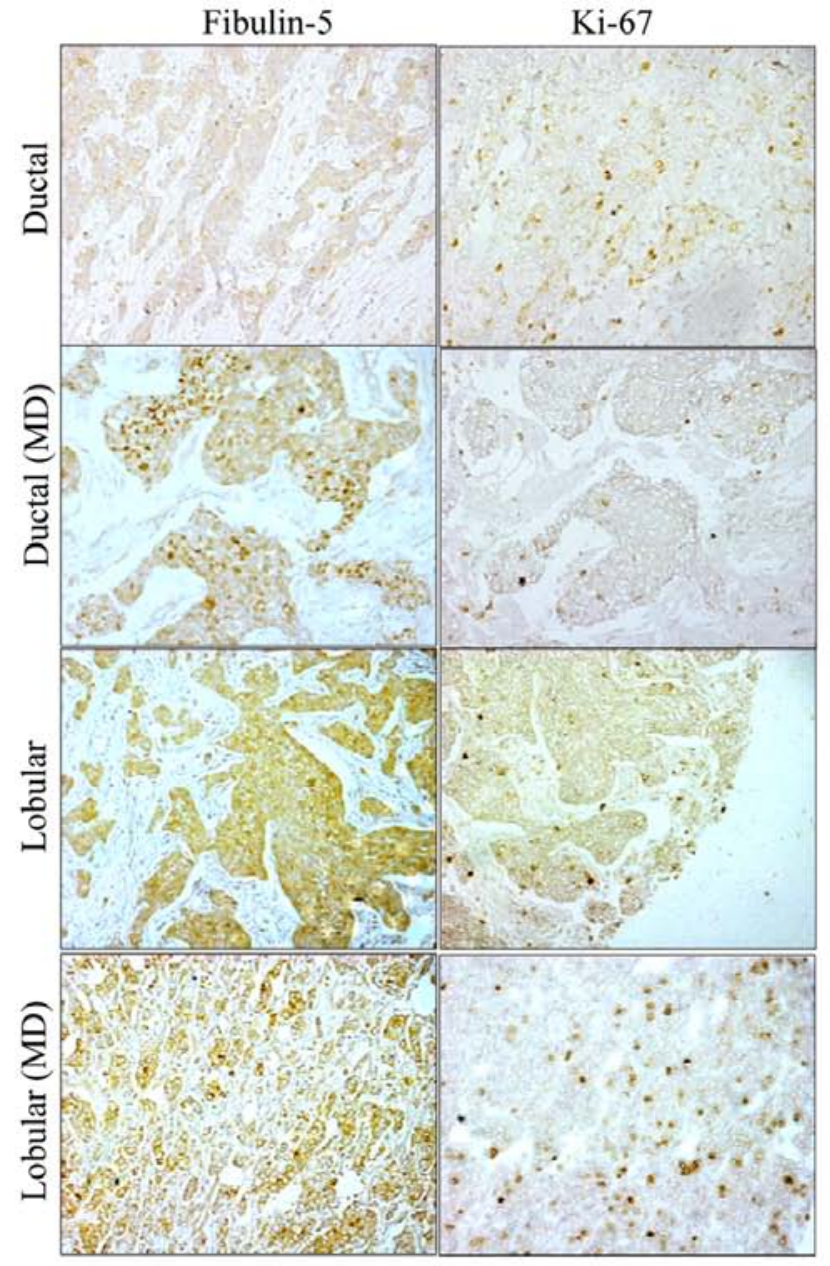
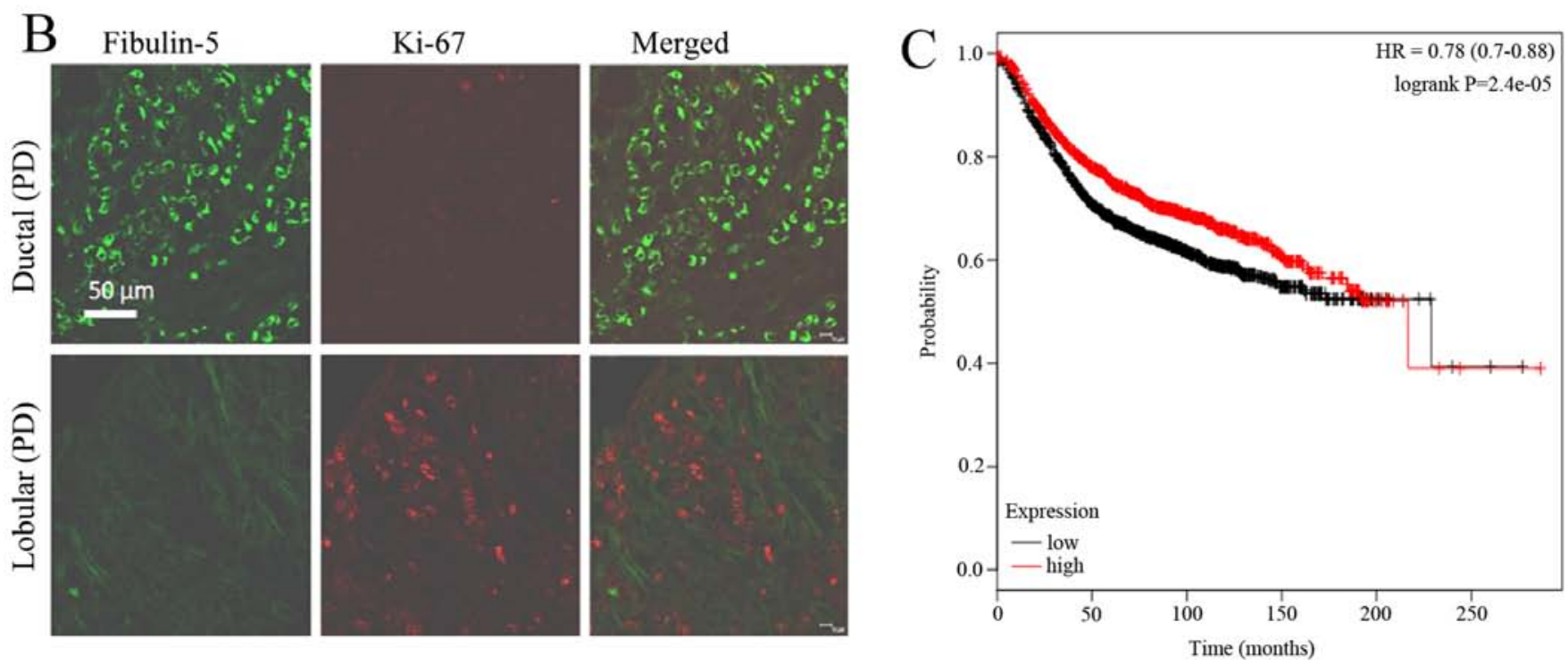

Figure 7. Fibulin-5 and Ki-67 expression analysis in breast cancer samples. (A) Representative tissue images for detection of Fibulin-5 and Ki-67 in normal and breast carcinoma samples including medullary, coloid, ductal and lobullar carcinoma (MD, moderately differentiated). Scale bar, $100 \mu \mathrm{m}$. (B) Laserconfocal double fluorescence for simultaneous detection of Fibulin-5 and Ki-67 in poorly differentiated (PD) ductal and lobular carcinomas. Scale bar, $50 \mu \mathrm{m}$. (C) Kaplan-Meier survival plots showing that high expression level of FBLN5 is associated to better outcome in breast cancer patients.

or tumor-suppressive effects depending on multiple factors, and Fibulin-5 is not an exception. For instance, the interaction between Fibulin- 5 and $\beta 1$ integrins regulates the levels of reactive oxygen species (ROS) in tumors, which has been shown to be increased in Fbln $5^{(--)}$mice in comparison with the wild-type littermates (28). Nogo-B, an isoform of reticulon-4, is also an interacting partner of Fibulin-5 and the consequence of this interaction is induction of EMT as well as the promotion of cell migration and invasion of $\mathrm{HeLa}$ cervical cancer cells (14). Fibulin-5 also enhances EMT and 
stimulates dedifferentiation of mammary epithelial cells upon induction by TGF- $\beta$ (13). Interestingly, FBLN5 is a TGF- $\beta$ inducible gene that can modulate protein kinase activities thus affecting cell growth and motility.

As opposed to these tumor-promoting effects, Fibulin-5 can also support tumor-protective properties (4). Thus, Fibulin-5 has been identified as angiostatic agent in tumors produced by MCA102 (19) and HT1080 (18) fibrosarcoma cells. Moreover, it has been found that Fibulin-5 hampers invasion and proliferation of human bladder, ovarian and lung cancer cells $(15,17,29)$, thereby inhibiting tumor growth. In the present study and using different cell-based assays we have found that Fibulin-5 also induces antitumor effects in three well-known human breast cancer cell lines. In fact, overexpression of Fibulin-5 decreases invasion of the poorly invasive T47D and MCF-7 cells, and also of the highly invasive MDA-MB-231 cells. Moreover, MDA-MB-231 cells overexpressing Fibulin-5 significantly reduce their in vitro migration capacity on type-I collagen. Interestingly, deposition of type-I collagen facilitates metastasis of breast cancer cells (30). Collectively, our data allow to speculate that the presence of Fibulin-5 may exert its antitumor effects through the modulation of a signaling pathway involved in breast cancer progression. Recently Chen et al (17) found that Fibulin-5 affects Wnt/ $\beta$-catenin signaling pathway in lung cancer cells. To evaluate the possibility that $\beta$-catenin is also affected in breast cancer cells upon overexpression of Fibulin-5, we explored the phosphorylation status in MCF-7 cells of the two forms $\beta$-catenin involved in its nuclear translocation. We found that phosphorylation levels of $\operatorname{Ser}^{675}$ and particularly of Ser ${ }^{552}$ were drastically reduced in MCF-7 cells overexpressing Fibulin-5 in comparison to control cells. Nuclear translocation of $\beta$-catenin implies an increase of its transcriptional activity which contributes to promote tumor metastasis not only in breast cancer cells, including MCF-7 (31-33), but also in different types of cancer (34). Consequently, Fibulin-5 could function as a tumor suppressor by blocking phosphorylation of $\beta$-catenin in breast cancer.

Two more findings highlight the antitumor effect displayed by Fibulin-5 in breast cancer cells. First, presence of Fibulin- 5 affects the capacity of tumor cells to form mammospheres. This effect strongly suggests that Fibulin-5 abrogates the ability of breast cancer cells for in vitro self-renewal (24). Second finding is that Fibulin-5 inhibits proliferation of breast tumors cells. Moreover, our data indicate that Fibulin-5 is associated with a low expression of $\mathrm{Ki}-67$, a nuclear protein related to cell proliferation and an important parameter in breast cancer prognosis (21). To explore if this effect can be reproduced in breast cancer samples we performed and immunohistochemical analysis. Detection of Fibulin-5 in breast cancer samples has been previously reported by Lee et al (13). These authors found that breast tumors contained abundant levels of Fibulin-5 protein despite the FBLN5 messenger RNA levels were reduced in mammary tumors attending to the data obtained from Oncomine Research Database. Our immunohistochemical study confirmed that Fibulin-5 is detected in breast cancer samples, and also revealed that its detection is concomitant with low detection of Ki-67. Moreover, ductal and lobular carcinoma, the two most common types of breast tumors, have a contrary evolution as poorly differentiated ductal carcinoma showed high Fibulin-5 and low Ki-67 detec- tion and vice versa. Overall, these data suggest that Fibulin-5 could influence breast cancer cell proliferation. Previous studies have addressed the influence of Fibulin-5 in cell proliferation and again, Fibulin- 5 showed a dual role as it can display both pro- and anti-proliferative effects. For instance, Fibulin-5 overexpression inhibits endothelial cell proliferation $(35,36)$. Moreover, Spencer et al (37) highlighted the role of Fibulin-5 as an inhibitor of vascular smooth muscle cell proliferation employing the Fbln5-deficient mice. By contrast, Fibulin-5 can also exert pro-proliferative effects in nasopharyngeal carcinoma cells (11). Based on these data, we speculate that upon secretion, Fibulin-5 can modify the local cell microenvironment through distinct interactions or establishing complex networks with other ECM that could either promote or repress a tumor microenvironment. In the present study we show new insights into the tumor-protective functions of Fibulin-5. Moreover, high expression levels of FBLN5 patients indicate a better outcome of breast cancer patients. Overall, these data could help to develop new therapeutic approaches to target tumor microenvironment.

\section{Acknowledgements}

We thank Dr Carlos López-Otín (Universidad de Oviedo) for providing cell lines and reagents, and Dr William P. Schiemann (Case Western Reserve University, Cleveland, $\mathrm{OH}$, USA) for providing plasmid containing cDNA for Fibulin-5. Y.M. is supported by a Ficyt (Gobierno del Principado de Asturias, Spain) fellowship and T.F. is supported by the IUOPA. The present study is partially supported by a grant from European Union FEDER funds, Principado de Asturias (Plan de Ciencia, Tecnologia e Innovacion), FICYT (GRUPIN 14-069), to J.A.V., S.C. and A.J.O, and by a grant from the Fondo de Investigaciones Sanitarias, FISS PI11/00371. IUOPA is supported by the Obra Social Cajastur, Asturias, Spain.

\section{References}

1. de Vega S, Iwamoto T and Yamada Y: Fibulins: Multiple roles in matrix structures and tissue functions. Cell Mol Life Sci 66: 1890-1902, 2009.

2. Yanagisawa $\mathrm{H}$ and Davis EC: Unraveling the mechanism of elastic fiber assembly: The roles of short fibulins. Int J Biochem Cell Biol 42: 1084-1093, 2010.

3. Papke CL and Yanagisawa H: Fibulin-4 and fibulin-5 in elastogenesis and beyond: Insights from mouse and human studies. Matrix Biol 37: 142-149, 2014.

4. Albig AR and Schiemann WP: Fibulin-5 function during tumorigenesis. Future Oncol 1: 23-35, 2005.

5. Lomas AC, Mellody KT, Freeman LJ, Bax DV, Shuttleworth CA and Kielty CM: Fibulin-5 binds human smooth-muscle cells through alpha5beta1 and alpha4betal integrins, but does not support receptor activation. Biochem J 405: 417-428, 2007.

6. Yanagisawa H, Davis EC, Starcher BC, Ouchi T, Yanagisawa M, Richardson JA and Olson EN: Fibulin-5 is an elastin-binding protein essential for elastic fibre development in vivo. Nature 415: 168-171, 2002.

7. Stone EM, Braun TA, Russell SR, Kuehn MH, Lotery AJ, Moore PA, Eastman CG, Casavant TL and Sheffield VC: Missense variations in the fibulin 5 gene and age-related macular degeneration. N Engl J Med 351: 346-353, 2004.

8. Jones RP, Ridley C, Jowitt TA, Wang MC, Howard M, Bobola N, Wang T, Bishop PN, Kielty CM, Baldock C, et al: Structural effects of fibulin 5 missense mutations associated with agerelated macular degeneration and cutis laxa. Invest Ophthalmol Vis Sci 51: 2356-2362, 2010. 
9. Gallagher WM, Currid CA and Whelan LC: Fibulins and cancer: Friend or foe? Trends Mol Med 11: 336-340, 2005.

10. Obaya AJ, Rua S, Moncada-Pazos A and Cal S: The dual role of fibulins in tumorigenesis. Cancer Lett 325: 132-138, 2012.

11. Hwang CF, Shiu LY, Su LJ, Yu-Fang Yin, Wang WS, Huang SC Chiu TJ, Huang CC, Zhen YY, Tsai HT, et al: Oncogenic fibulin-5 promotes nasopharyngeal carcinoma cell metastasis through the FLJ10540/AKT pathway and correlates with poor prognosis. PLoS One 8: e84218, 2013.

12. Ohara H, Akatsuka S, Nagai H, Liu YT, Jiang L, Okazaki Y, Yamashita Y, Nakamura T and Toyokuni S: Stage-specific roles of fibulin-5 during oxidative stress-induced renal carcinogenesis in rats. Free Radic Res 45: 211-220, 2011.

13. Lee YH, Albig AR, Regner M, Schiemann BJ and Schiemann WP Fibulin-5 initiates epithelial-mesenchymal transition (EMT) and enhances EMT induced by TGF-beta in mammary epithelial cells via a MMP-dependent mechanism. Carcinogenesis 29 : 2243-2251, 2008.

14. Xiao W, Zhou S, Xu H, Li H, He G, Liu Y and Qi Y: Nogo-B promotes the epithelial-mesenchymal transition in HeLa cervical cancer cells via Fibulin-5. Oncol Rep 29: 109-116, 2013.

15. Hu Z, Ai Q, Xu H, Ma X, Li HZ, Shi TP, Wang C, Gong DJ and Zhang X: Fibulin-5 is down-regulated in urothelial carcinoma of bladder and inhibits growth and invasion of human bladder cancer cell line 5637. Urol Oncol 29: 430-435, 2011.

16. Yue W, Sun Q, Landreneau R, Wu C, Siegfried JM, Yu J and Zhang L: Fibulin-5 suppresses lung cancer invasion by inhibiting matrix metalloproteinase-7 expression. Cancer Res 69: 6339-6346, 2009.

17. Chen X, Song X, Yue W, Chen D, Yu J, Yao Z and Zhang L: Fibulin-5 inhibits Wnt $/ \beta$-catenin signaling in lung cancer. Oncotarget 6: 15022-15034, 2015.

18. Xie L, Palmsten K, MacDonald B, Kieran MW, Potenta S, Vong S and Kalluri R: Basement membrane derived fibulin-1 and fibulin-5 function as angiogenesis inhibitors and suppress tumor growth. Exp Biol Med (Maywood) 233: 155-162, 2008.

19. Albig AR, Neil JR and Schiemann WP: Fibulins 3 and 5 antagonize tumor angiogenesis in vivo. Cancer Res 66: 2621-2629, 2006.

20. Wlazlinski A, Engers R, Hoffmann MJ, Hader C, Jung V, Müller M and Schulz WA: Downregulation of several fibulin genes in prostate cancer. Prostate 67: 1770-1780, 2007.

21. Inwald EC, Klinkhammer-Schalke M, Hofstädter F, Zeman F, Koller M, Gerstenhauer M and Ortmann O: $\mathrm{Ki}-67$ is a prognostic parameter in breast cancer patients: Results of a large populationbased cohort of a cancer registry. Breast Cancer Res Treat 139: 539-552, 2013

22. Schiemann WP, Blobe GC, Kalume DE, Pandey A and Lodish HF: Context-specific effects of fibulin-5 (DANCE/ EVEC) on cell proliferation, motility, and invasion. Fibulin-5 is induced by transforming growth factor-beta and affects protein kinase cascades. J Biol Chem 277: 27367-27377, 2002.

23. Rhodes LV, Antoon JW, Muir SE, Elliott S, Beckman BS and Burow ME: Effects of human mesenchymal stem cells on ER-positive human breast carcinoma cells mediated through ER-SDF-1/CXCR4 crosstalk. Mol Cancer 9: 295, 2010.

24. Dontu G, Abdallah WM, Foley JM, Jackson KW, Clarke MF, Kawamura MJ and Wicha MS: In vitro propagation and transcriptional profiling of human mammary stem/progenitor cells. Genes Dev 17: 1253-1270, 2003.
25. Győrffy B, Benke Z, Lánczky A, Balázs B, Szállási Z, Timár J and Schäfer R: RecurrenceOnline: An online analysis tool to determine breast cancer recurrence and hormone receptor status using microarray data. Breast Cancer Res Treat 132: 1025-1034, 2012 .

26. Manuel Iglesias J, Beloqui I, Garcia-Garcia F, Leis O, VazquezMartin A, Eguiara A, Cufi S, Pavon A, Menendez JA, Dopazo J, et al: Mammosphere formation in breast carcinoma cell lines depends upon expression of E-cadherin. PLoS One 8: e77281, 2013.

27. Kelly T, Yan Y, Osborne RL, Athota AB, Rozypal TL, Colclasure JC and Chu WS: Proteolysis of extracellular matrix by invadopodia facilitates human breast cancer cell invasion and is mediated by matrix metalloproteinases. Clin Exp Metastasis 16: $501-512,1998$.

28. Schluterman MK, Chapman SL, Korpanty G, Ozumi K, Fukai T, Yanagisawa $\mathrm{H}$ and Brekken RA: Loss of fibulin-5 binding to betal integrins inhibits tumor growth by increasing the level of ROS. Dis Model Mech 3: 333-342, 2010.

29. Heo JH, Song JY, Jeong JY, Kim G, Kim TH, Kang H, Kwon AY and An HJ: Fibulin-5 is a tumour suppressor inhibiting cell migration and invasion in ovarian cancer. J Clin Pathol: jclinpath2015-203129, 2015.

30. Zhang K, Corsa CA, Ponik SM, Prior JL, Piwnica-Worms D, Eliceiri KW, Keely PJ and Longmore GD: The collagen receptor discoidin domain receptor 2 stabilizes SNAIL1 to facilitate breast cancer metastasis. Nat Cell Biol 15: 677-687, 2013.

31. Cai J, Guan H, Fang L, Yang Y, Zhu X, Yuan J, Wu J and Li M: MicroRNA-374a activates Wnt/ $\beta$-catenin signaling to promote breast cancer metastasis. J Clin Invest 123: 566-579, 2013.

32. Klemm F, Bleckmann A, Siam L, Chuang HN, Rietkötter E, Behme D, Schulz M, Schaffrinski M, Schindler S, Trümper L, et al: $\beta$-catenin-independent WNT signaling in basal-like breast cancer and brain metastasis. Carcinogenesis 32: 434-442, 2011.

33. Laezza C, D'Alessandro A, Paladino S, Maria Malfitano A, Chiara Proto M, Gazzerro P, Pisanti S, Santoro A, Ciaglia E and Bifulco M; Endocannabinoid Research Group: Anandamide inhibits the Wnt/ $\beta$-catenin signalling pathway in human breast cancer MDA MB 231 cells. Eur J Cancer 48: 3112-3122, 2012.

34. Fang D, Hawke D, Zheng Y, Xia Y, Meisenhelder J, Nika H, Mills GB, Kobayashi R, Hunter T and Lu Z: Phosphorylation of beta-catenin by AKT promotes beta-catenin transcriptional activity. J Biol Chem 282: 11221-11229, 2007.

35. Preis M, Cohen T, Sarnatzki Y, Ben Yosef Y, Schneiderman J, Gluzman Z, Koren B, Lewis BS, Shaul Y and Flugelman MY: Effects of fibulin-5 on attachment, adhesion, and proliferation of primary human endothelial cells. Biochem Biophys Res Commun 348: 1024-1033, 2006.

36. Li F, Xu H, Zeng Y and Yin ZQ: Overexpression of fibulin-5 in retinal pigment epithelial cells inhibits cell proliferation and migration and downregulates VEGF, CXCR4, and TGFB1 expression in cocultured choroidal endothelial cells. Curr Eye Res 37: 540-548, 2012.

37. Spencer JA, Hacker SL, Davis EC, Mecham RP, Knutsen RH, Li DY, Gerard RD, Richardson JA, Olson EN and Yanagisawa H: Altered vascular remodeling in fibulin-5-deficient mice reveals a role of fibulin-5 in smooth muscle cell proliferation and migration. Proc Natl Acad Sci USA 102: 2946-2951, 2005. 